\title{
RESEARCH HIGHLIGHT OPEN SARS-CoV-2 variants: a new challenge to convalescent serum and mRNA vaccine neutralization efficiency
}

\author{
Maochen Li (D) ${ }^{1}$, Fuxing Lou $^{1}$ and Huahao Fan (D) \\ Signal Transduction and Targeted Therapy (2021)6:151
}

The spike protein of SARS-CoV-2 is the target of antibodies in convalescent and vaccine sera, and 23 mutations in spike protein were reported in the variants B.1.1.7, B.1.351, and P.1 (Fig. 1a). Recently, several groups evaluated the effects of convalescent and mRNA vaccine sera on two major circulating SARS-CoV-2 variants B.1.1.7 and B.1.351 (Fig. 1b), ${ }^{1-5}$ which leads to concerns about the immune escape of these variants from the human acquired immunity stimulated by previous infections and mRNA vaccines.

By 27 March 2021, COVID-19 has caused more than 126.1 million infections and 2,768,409 deaths (https://coronavirus.jhu. edu/), and some countries have already developed vaccines against SARS-CoV-2. Different from the inactivated vaccines approved in China, the western countries are prone to develop mRNA vaccines or viral vector vaccines (e.g., Oxford-AstraZeneca vaccine ChAdOx1 nCoV-19 (AZD1222) and Pfizer-BioNTech vaccine BNT162b2), targeting the spike protein, whose mutations deserve consistent monitoring. Because of the instability of SARSCoV-2 RNA and error-prone replication, viral mutations appear frequently. ${ }^{1,2}$ Until now, 114, 67, and 36 countries have reported the discovery of variants B.1.1.7, B.1.351, and P.1, respectively (covlineages.org/global_report.html). Given these mutations may cause higher viral load and longer infection duration in the infected persons, ${ }^{3}$ the effects of B.1.1.7 and B.1.351 variants on virus infection and vaccine efficiency were studied by several groups, respectively (Fig. 1b). ${ }^{1,2,4,5}$

Supasa, P. et al. analyzed 180,000 sequences from the COG-UK database (https://www.cogconsortium.uk) and found that B.1.1.7 strain with amino acid 69 and 70 of spike protein deletion $(\Delta 69 / 70)$ occupied the dominant position among the three subgroups (the other subgroups lack this deletion), which indicated selective advantages existed in the variation process of SARS-CoV-2. ${ }^{1}$ And N501Y mutation existing in all B.1.1.7, B.1.351, and P.1 variants were found to enhance the affinity between receptor-binding domain (RBD) and angiotensin-converting enzyme 2 (ACE2) by about 7-fold compared with wild type (WT). According to the data released by Public Health England, the infectivity of B.1.1.7 variant is $30-50 \%$ higher than that of the wild type (WT), which may result from the increased affinity between RBD and ACE2. ${ }^{3}$ Then the neutralization activities of 20 potent monoclonal antibodies for WT were detected by focus reduction neutralization tests, and a remarkable neutralization activity reduction was found in IGHV3-53 mAbs (e.g., mAb269 almost completely lost neutralization activity and mAb278 only retained $78 \%$ activity at most). In addition, the authors tested the efficacy of neutralizing antibodies in vaccine sera. The vaccine

\author{
; https://doi.org/10.1038/s41392-021-00592-6
}

AZD1222 sera at 14 and 28 days following the second dose, and the vaccine BNT162b2 sera at 7-17 days following the second dose were tested, and the neutralizing activity of vaccine AZD1222 and BNT162b2 against the B.1.1.7 variant decreased by 2.5-fold and 3.3-fold, respectively. However, the cocktail therapy is still effective for neutralizing B.1.1.7 variant. Moreover, the convalescent sera from 13 B.1.1.7 patients can neutralize both B.1.1.7 variant and Victoria variant, indicating the potential of B.1.1.7 as the seed strain for future inactivated vaccines. Given the above information, the author hold the opinion that the current vaccines being arranged to massive scales against COVID-19 is still robust and no obvious evidence is found for immune escape. ${ }^{1}$

Almost at the same time, Peiyong Shi and colleagues estimated the neutralizing efficiency of BNT162b2 vaccine sera on N501Y mutants and B.1.351 variants. ${ }^{4,5}$ Based on the SARS-CoV-2 reverse genetic system developed previously, they obtained the variants using the SARS-CoV-2 USA-WA1/2020 strain, a isolate identified in January 2020: (1) N501Y; (2) $\Delta 69 / 70$ + N501Y + D614G; (3) E484K + N501Y + D614G; (4) $\Delta 242-244$ + D614G; (5) K417N + E484K + $\mathrm{N} 501 Y$ + D614G; (6) B.1.351-spike. ${ }^{4,5}$ The ratios of the neutralization geometric mean titers (GMTs) of the sera against above mutants to their GMTs against the USA-WA1/2020 virus were 1.46, $1.41,0.81,0.97,0.66$, and 0.37 , respectively (Fig. $1 \mathrm{~b}$ ), which again implied that E484 and K417 are key sites for virus immune escape. ${ }^{4,5}$ Due to the continuous mutations of SARS-CoV-2 spike protein, mRNA vaccines might fail to have the potent efficiency as previously expected, suggesting viable methods (for instance, cocktail mRNA vaccines) are needed to be proposed to develop the effective mRNA vaccine.

Thomson et al. found that receptor-binding motif (RBM) sequence is a highly variable region, which contained N501Y mutation in both variants B.1.1.7 and B.1.351 mentioned above. ${ }^{2}$ And another mutation $\mathrm{N} 439 \mathrm{~K}$, the second most prevalent mutation of spike protein also located in the RBM sequence (Fig. 1a), has been predicted to cause about 2-15 million infections worldwide according to a statistical model. ${ }^{2} \mathrm{~N} 439 \mathrm{~K}$ has been deemed to increase the viral load about 1.54-fold in 1918 Scottish patients, owing to the higher affinity between RBD and hACE2. ${ }^{2}$ Then, the recognition of N439K RBD by immune serum from 442 recovered individuals ( 6 patients were infected by $\mathrm{N} 439 \mathrm{~K}$ mutants) as well as 140 monoclonal antibodies from COVID-19 patients (including REGN-CoV, which obtained the EUA of FDA) were evaluated, $6.8 \%$ of the serum samples and $16.7 \%$ of mAbs had more than 2-fold reduction in binding activity, respectively. Interestingly, a camelid nanobody $\mathrm{VHH}-72$ showed

\footnotetext{
${ }^{1}$ Beijing Advanced Innovation Center for Soft Matter Science and Engineering, College of Life Science and Technology, Beijing University of Chemical Technology, Beijing, China Correspondence: Huahao Fan (fanhuahao@mail.buct.edu.cn)

These authors contributed equally: Maochen Li, Fuxing Lou
}

Received: 27 February 2021 Revised: 27 March 2021 Accepted: 29 March 2021

Published online: 10 April 2021 
a

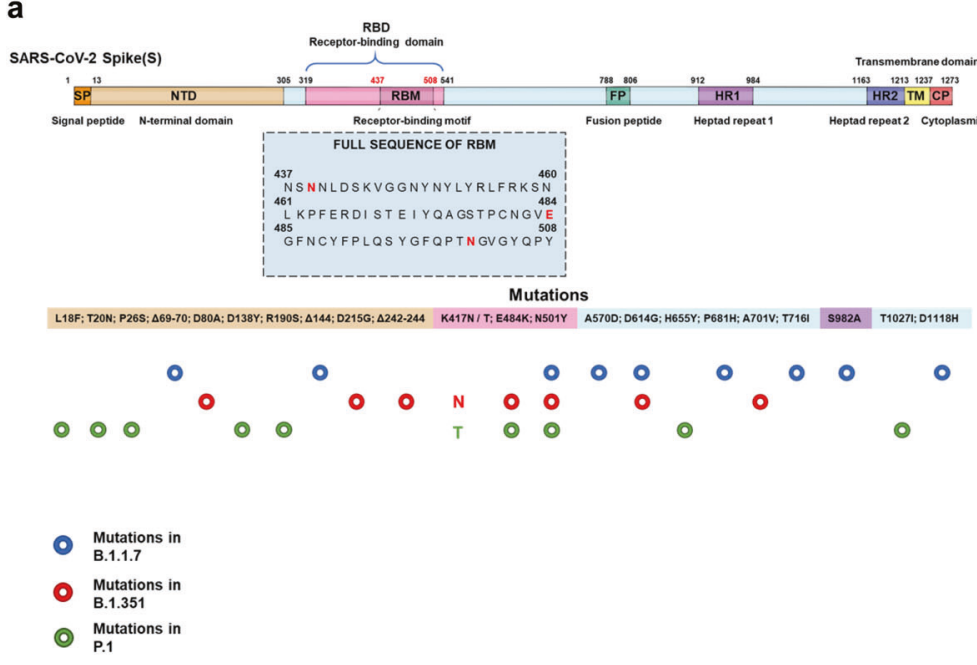

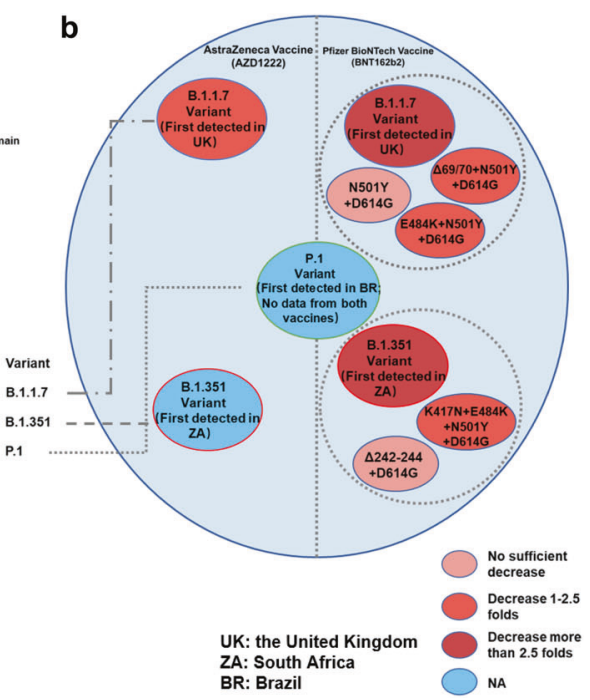

Fig. 1 A schematic illustration of SARS-CoV-2 S protein sequence, prevalent mutations in current circulating SARS-CoV-2 variants, and the neutralization efficiency change of two mRNA vaccines-vaccine AZD1222 and vaccine BNT162b2. a RBM sequence (amino acid 437-508) containing N439K, E484K, and N501Y is a highly variable region, and SARS-CoV-2 spike protein mutations of three major circulating variants B.1.1.7, B.1.351, and P.1 are displayed, which can be tracked online (https://covariants.org/, https://cov-lineages.org/). b The neutralization efficiency of two major vaccines-vaccine AZD1222 and vaccine BNT162b2 to major circulating variants and potentially important variants of SARS-CoV-2 are exhibited. The neutralizing activity of the vaccine sera against different variants are shown in different colors from light red (no sufficient decrease), lycopene (decrease between 1.5 -fold and 2.5-fold), to wine red (decrease more than 2.5-fold)

an increased neutralization on $\mathrm{N} 439 \mathrm{~K}$ variant, suggesting the mutation may be a double-edged sword for maintaining fitness of the $\mathrm{N} 439 \mathrm{~K}$ variants. ${ }^{2}$

In summary, these recent studies evaluated the major circulating SARS-CoV-2 variants B.1.1.7 and B.1.351 by convalescent and vaccine sera, and highlighted the importance of continuous SARSCoV-2 molecular surveillance. Meantime, these researches are significant for the guidance of COVID-19 therapy, vaccine redesigning, and epidemic prevention: (1) For the COVID-19 patients, a personalized COVID-19 antibody therapy or cocktail therapy will be beneficial from the local circulating variants screening; (2) Desirable vaccine candidates, multivalent vaccines or cocktail vaccines should be designed to neutralize all circulating variants; (3) Inactivated vaccine seed strain of circulating SARSCoV-2 variants is worthy of development for future epidemic prevention.

\section{ACKNOWLEDGEMENTS}

We thank Prof. Yigang Tong and Lihua Song (Beijing University of Chemical Technology, China) for helpful discussions and valuable suggestions. Huahao Fan declare grants from National Key Research and Development Program of China (grant No. 2020YFA0712102), Fundamental Research Funds for Central Universities (grant No. BUCTZY2022), and H\&H Global Research and Technology Center (grant No. H2021028).

\section{AUTHOR CONTRIBUTIONS}

H.F. designed the research; H.F., M.L., and F.L. read the papers and analyzed the data; H.F. wrote and revised the manuscript.

\section{ADDITIONAL INFORMATION}

Competing interests: The authors declare no competing interests.

\section{REFERENCES}

1. Supasa, P. et al. Reduced neutralization of SARS-CoV-2 B.1.1.7 variant by convalescent and vaccine sera. Cell 184, 1-11 (2021).

2. Thomson, E. C. et al. Circulating SARS-CoV-2 spike N439K variants maintain fitness while evading antibody-mediated immunity. Cell 184, 1171-1187.e20 (2021).

3. Grubaugh, N. D. et al. Public health actions to control new SARS-CoV-2 variants. Cell 184, 1127-1132 (2021).

4. Xie, X. et al. Neutralization of SARS-CoV-2 spike $69 / 70$ deletion, E484K and N501Y variants by BNT162b2 vaccine-elicited sera. Nat. Med. https://doi.org/10.1038/ s41591-021-01270-4 (2021)

5. Liu, Y. et al. Neutralizing Activity of BNT162b2-Elicited Serum-Preliminary Report. NEJM https://doi.org/10.1056/NEJMc2102017 (2021).

(C) Open Access This article is licensed under a Creative Commons Attribution 4.0 International License, which permits use, sharing, adaptation, distribution and reproduction in any medium or format, as long as you give appropriate credit to the original author(s) and the source, provide a link to the Creative Commons license, and indicate if changes were made. The images or other third party material in this article are included in the article's Creative Commons license, unless indicated otherwise in a credit line to the material. If material is not included in the article's Creative Commons license and your intended use is not permitted by statutory regulation or exceeds the permitted use, you will need to obtain permission directly from the copyright holder. To view a copy of this license, visit http://creativecommons. org/licenses/by/4.0/.

(c) The Author(s) 2021 


\section{QUERY FORM}

\section{SIGTRANS}

\begin{tabular}{|l|l|}
\hline Manuscript ID & [Art. Id: 592] \\
\hline Author & \\
\hline Editor & \\
\hline Publisher & \\
\hline
\end{tabular}

\section{Journal: SIGTRANS}

Author :- The following queries have arisen during the editing of your manuscript. Please answer by making the requisite corrections directly in the e.proofing tool rather than marking them up on the PDF. This will ensure that your corrections are incorporated accurately and that your paper is published as quickly as possible.

\begin{tabular}{|c|l|l|}
\hline $\begin{array}{c}\text { Query } \\
\text { No. }\end{array}$ & Description & Author's Response \\
\hline & No queries & \\
\hline
\end{tabular}

